\title{
Study on the Distribution Law of Front Abutment Pressure of Long Fully-Mechanized Working Face in Deep Mine
}

\author{
Yuguo Ji, Xianjun Wang, Yongpei Zhou, Xiantang Zhang \\ Shandong University of Science and Technology line 3- Qingdao 266590, China \\ 736562385@qq.com
}

\begin{abstract}
The front abutment pressure of long fullymechanized working face has an important influence on the law of rock motion and emergence of rock burst, and it is also directly related to safety and efficiency of production. Based on a fully-mechanized working face, by using Fast Language Analysis of continua in 2 dimensions (FLAC2D), the authors carried study on the distribution law of front abutment pressure by theoretical calculation and numerical simulation. According to the results, the influenced area can be divided into two parts: one is the plastic area, where the abutment pressure grows fast in the form of exponential function from working face to the inside, and the abutment pressure reaches to peak value with certain range. The other one is elastic area, in which the abutment pressure firstly decreases in a fast speed and it transits to initial rock stress finally.
\end{abstract}

Keywords - abutment pressure, working face, FLAC2D, numerical simulation, plastic area.

\section{INTRODUCTION}

Coal is the main energy source of our country, and the demand of it is increasing day by day with the rapid economic development. Many mines in shallow-buried strata were exploited and coal mining now turns to deep-buried strata ${ }^{1}$, meanwhile, comprehensive mining technologies and equipment have developed well in coal industry so that the length of working face is much longer than before. The ground stress in deep mine is relatively high, thus the redistribution of stress, which is called abutment pressure in front of the working face, can be more complicated. The complicated abutment pressure usually has direct influence on increased support load that is induced by the immediate roof and rock motion, degree of roof breakage in roof-control area ${ }^{2}$, and the frequency and intensity of rock burst in working face ${ }^{3,4}$. Therefore, it is very valuable to master the law of pressure distribution of fully mechanized mining face in deep mine, which can help improve safety and efficiency of production and reduce sudden danger. Many scholars carried out study on the distribution law of front abutment pressure of working face. Some of them carried out study based on damage mechanics ${ }^{5}$, and then, some made their research on abutment pressure based on certain mining thickness, mining height and width of coal pillar-9, also, scholars studied the abutment pressure in the fully-mechanized working face of isolated island by numerical simulation ${ }^{10-12}$. Besides, based on the characteristic of micro-seismic events, some scholars studied the apparent stress and abutment pressure by waveform analysis and inversion of the micro-seismic activity ${ }^{13}$.

\section{MATERIALS AND METHODS}

The study related to the front abutment pressure of long fully-mechanized working face at the thousands of meters is relatively rare. Therefore, in this paper, based on the condition of NO.7435 working face, the VI1 mining area of Kongzhuang Coal Mine, Datun coal and electricity company, authors carried out study on the front abutment pressure of it by theoretical calculation and numerical simulation, using FLAC $^{2 D}$ to find the law of abutment pressure of this working face ${ }^{14,15}$, hoping to provide technical reference for the field production and similar working faces.

\section{A. Theoretical calculation of front abutment pressure}

\section{1) Calculation of plastic area:}

When the mining depth exceeds a certain value, the stress value is higher than the strength of the coal, at this moment, the coal wall without support will bear large vertical pressure, and the brittle failure caused by compressive and tensile action, similar to the damage under uniaxial compression, will emerge, which is also called rib spalling. Then, the side constraint of the area that is in front of the destroyed area bearing compressive stress increases a lot, but considering the deep mining depth, the vertical stress is still much larger than lateral stress, thus shear failure emerges in certain area, which is also considered as plastic area. After that, the elastic area appears. Therefore, under the effect of abutment pressure, the front area of working face can be divided into three parts: brittle failure area caused by compressive and tensile action, plastic area and elastic deformation area ${ }^{16}$. In order to simplify the calculation, brittle failure area is also considered as plastic area in the theoretical calculation and numerical simulation. According to the elastoplastic theory and the formula of the abutment pressure in the limit equilibrium area in front of the working face ${ }^{7,17}$, the abutment pressure of plastic area $\sigma_{\mathrm{y}}$ is: 


$$
\sigma_{y}=\tau_{0} \cot \varphi \frac{1+\sin \varphi}{1-\sin \varphi} e^{\frac{2 f_{x}}{M}\left(\frac{1-\sin \varphi}{1+\sin \varphi}\right)}
$$

Where $f$ is friction coefficient between interlayer, $\tau_{0} \cot \varphi-$ the support force of coal, $\tau_{0}-$ shear stress, $x$ - distance from plastic area to coal wall, $M$ - thickness of coal seam, $\mathrm{m}$.

Let $\sigma_{\mathrm{y}}=K \cdot \gamma \cdot H$, and the distance from the peak value of abutment pressure to the coal wall $x_{0}$ is:

$$
x_{0}=\frac{M}{2 f}\left(\frac{1+\sin \varphi}{1-\sin \varphi}\right) \ln \left(\frac{K \gamma H}{\tau_{0}} \frac{(1-\sin \varphi)}{1+\sin \varphi}\right)
$$

Where, $K$ is stress concentration factor, $\gamma$ - bulk density of overlying strata, $\mathrm{KN} / \mathrm{m}^{3}, H$-depth of coal, $\mathrm{m}$.

In the relative length of mine exploitation of the same working face, the friction coefficient between interlayer can be assumed to be a constant, thus the peak value of front value is only connected to the thickness of coal, and the thicker the coal is, the larger the peak value is. Meanwhile, when the depth of the mine is deeper, the site that the peak value of front abutment pressure of working face emerges will move to the far site from the working face.

\section{2) Calculation of elastic area:}

After the appearance of shear failure in working face, the failure will move into the inside part of rock, but the strength of rock is better, and then the rock will transmit into elastic state, the formula of the abutment pressure in elastic area is ${ }^{17}$ :

$$
\sigma_{\mathrm{y}}=K \gamma H \mathrm{e}^{\frac{2 f}{\mathrm{~m} \beta}\left(x_{0}-x\right)}
$$

Where, $\beta$ is lateral pressure coefficient.

If the elastic deformation area is $x_{1}$, when $x=x_{0}+x_{1}$, the abutment pressure of this site is initial stress ${ }^{18}$, the value is: $\sigma_{\mathrm{y}}=\gamma H$

Formula (3) equals to formula (4), and put (4) into (3):

$x_{1}=\frac{M \beta}{2 f} \ln K$

According to formula (5), when the thickness of coal increases, the area that bears elastic abutment pressure in front of working face $x_{1}$ becomes larger. Based on the above analysis and formulas, formula (2) and (5) constitute the form of the front abutment pressure of working face, which is shown as Fig. 1. According to formula (2), during the plastic area, the abutment pressure is lower than initial stress, and the abutment pressure grows in the form of exponential function when the distance to coal wall is larger, and then, the pressure reaches the peak value $K \gamma H$ at the balanced site between plastic area and elastic area. According to formula (5), when the elastic area emerges, the stress concentration factor decreases gradually, and the abutment pressure decreases in the form of logarithmic function. When the stress concentration factor is 1 , the stress concentration phenomenon disappears, and the abutment transmits into initial stress $\gamma H$. Therefore, the influenced area of front abutment area pressure of working face becomes wider, with the growth of coal thickness and depth of mine. And the stress concentration factor $K$ becomes larger because of the superposition effect of abutment pressure ${ }^{18}$ According to the exploitation condition of NO.7435 and related calculation, the peak value of abutment pressure is $70.2 \mathrm{MPa}$, the distance between the working face to the site of peak value is $6.67 \mathrm{~m}$, and the distinct distance of the front area is $40.2 \mathrm{~m}$.

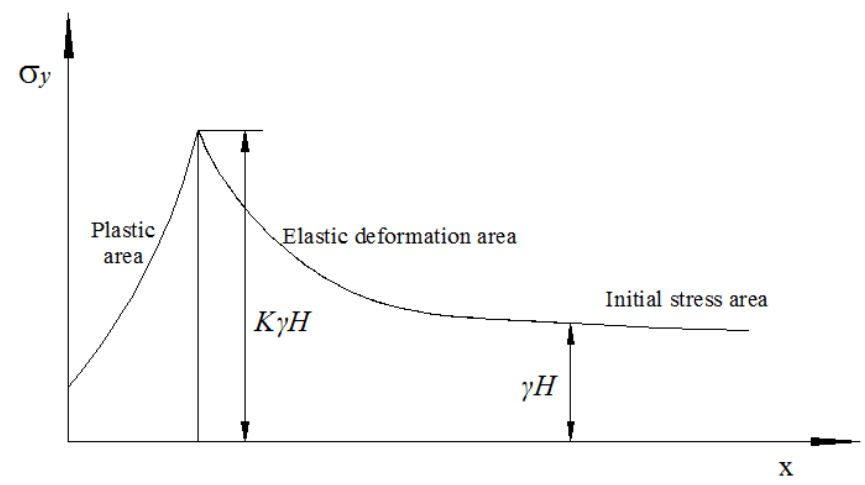

Fig. 1 supporting pressure area graph

\section{B. Numerical simulation of front abutment pressure}

The NO.7435 working face locates at the second track of IV1 mine area, the average dip angle of coal seam is $21^{\circ}$, the average thickness of coal is $4.6 \mathrm{~m}$, and the depth of mine is about $1000 \mathrm{~m}$. The roof rock is dark-grey sandy mudstone, which is sandy structure, smooth in fracture, plenty of plant fossil fragments and pyrite films, and is easy to fall. The coal seam floor is also sandy mudstone, dark grey, plenty of plant fossil fragments, and is smooth in facture. But the sand is not well-distributed.

\section{1) Numerical calculation model}

Based on the above geological conditions and the engineering practice, use the FLAC ${ }^{2 \mathrm{D}}$ to build simulation model of this working face ${ }^{19,20}$, the mechanical parameters of rock are in Table 1, and the stratigraphic distribution is shown in Fig. 2.In the model, the length is $700 \mathrm{~m}$, the width is $300 \mathrm{~m}$, the vertical direction is the depth of rock, and other 700-meter rock is applied in the form of equal and uniform load on the top boundary of model, and the value is $17.5 \mathrm{MPa}$. Set the side boundaries as lateral displacement boundary, the bottom boundary as vertical displacement boundary, top boundary as free boundary. And every grid is $1 \times 1 \mathrm{~m}$. 
TABLE1 Mechanical parameters of coal and rock

\begin{tabular}{|c|c|c|c|c|}
\hline rock & Bulk modulus (GPa) & $\begin{array}{l}\text { density } \\
\text { (kg/m3) }\end{array}$ & cohesion (MPa) & $\begin{array}{c}\text { internal friction } \\
\text { angle }(\rho)\end{array}$ \\
\hline Medium sand & 8.9 & 2600 & 12 & 40 \\
\hline Fine sandstone & 9.9 & 2700 & 15 & 40 \\
\hline Siltstone & 6.9 & 2800 & 7 & 30 \\
\hline Coal & 2.25 & 1400 & 4 & 20 \\
\hline Sandy mudstone & 6.5 & 2650 & 8 & 30 \\
\hline Mudstone & 6.9 & 2500 & 6 & 25 \\
\hline
\end{tabular}

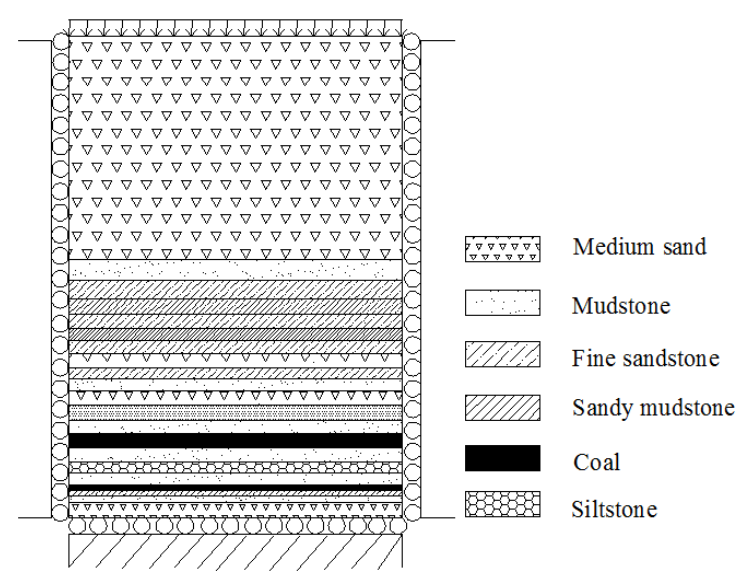

Fig. 2 The stratigraphic distribution

\section{2) Result and discussion}

After the calculation of the numerical model, the distribution of maximum principal stress is shown in Fig. 3, in which the stress concentration is pretty distinct.

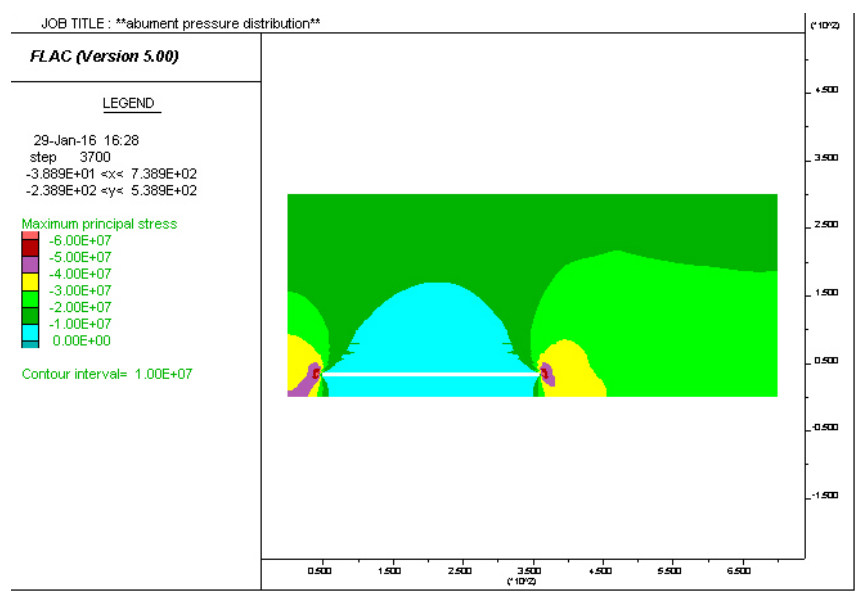

Fig. 3 The maximum principal stress

Fig. 4 is the distribution curve of the abutment pressure of working face form model, and it is very similar to the distribution of abutment pressure in Fig. 1. The distinctly influenced range is about $41 \mathrm{~m}$, the whole influenced range is about $200 \mathrm{~m}$, and the peak value is about $69.5 \mathrm{Mpa}$. The distance between peak and coal wall is $7 \mathrm{~m}$, and the stress concentration factor is 2.6. The results in simulation agree with that in theoretical calculation.

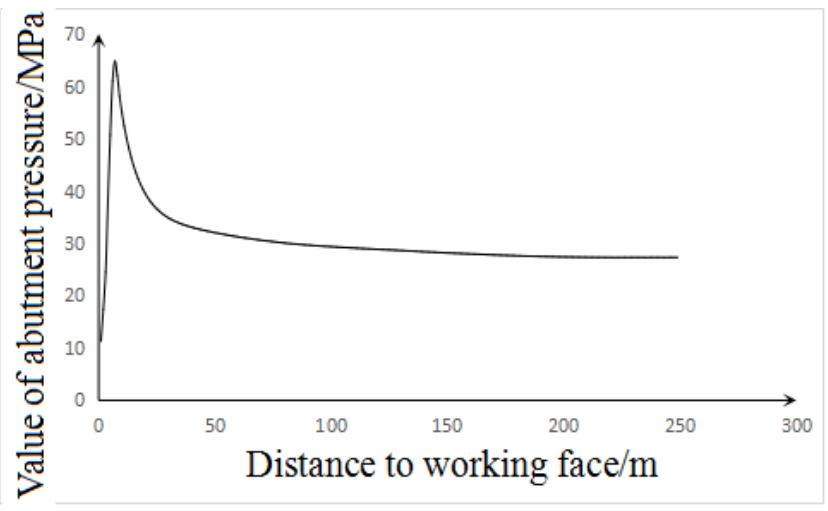

Fig. 4 The distribution curve of front abutment pressure

According to the curve, firstly, the stress value is lower than initial stress, but before the peak, the stress grows with a high speed. After the peak, with the growing distance to the coal wall, the variation of stresses can be described as two stats. In the first stage, the stress reduces with a high speed, similar to the logarithmic function. In the second stage, the stress reduces in a slow speed and finally it transmits into initial stress area. The second stage is relatively long both in time and range due to the pretty deep depth.

\section{CONCLUSIONS}

According to the theoretical calculation and numerical simulation, the results of the front abutment pressure of working face are basically consistent in variation tendency, peak value, distance between peak value to working face, and the conclusions are as follows:

1) During plastic area, the first range is the decreased stress area compared to the initial stress area and after that is 
the increased stress. Before the stress reaches the peak, it grows fast in the form of exponential function.

2) After the peak it transmits into elastic deformation area, the abutment pressure firstly decreases fast basically in the form of logarithmic function, and then it decreases slowly, the slow-decreasing range is relatively large due to the deep mine depth.

3) The distinctly influenced range of NO.7435 working face is about $41 \mathrm{~m}$, the peak value is about $69.5 \mathrm{Mpa}$, and the distance between peak and coal wall is about $7 \mathrm{~m}$.

\section{REFERENCES}

[1]Wang Qi. Research on control mechanism of surrounding rock failure in deep roadways with thick top-coal and contrast of new support systems. Ji Nan: Shan Dong University, 2012.

[2]Niu Zongjie, Hu Yaoqing, Wang Chenlong, Shao Mingwei. Influence of support system in roof - control area on relationship mechanics between surrounding rock and support. Safety in Coal Mines, 2014, 45(10):194197.

[3]Qian Qihu. Definition, mechanism, classification and quantitative forecast model for rockburst and pressure bump. Rock and Soil Mechanics, 2014, 35(1):1-6.

[4]Zhu Sitao, Feng Yu, Jiang Fuxing. Determination of abutment pressure in coal mines with extremely thick alluvium stratum: a typical kind of rockburst mines in China. Rock Mechnics and Rock Engineering, 2016, 49:1943-1952.

[5]Chen Zhonghui, Xie Heping. Damage mechanics analysis on the distribution of abutment pressure around a coal face. Chinese Journal of Rock Mechanics and Engineering, 2000, 19(4):436-439.

[6]Wang Zhen, Ou Cong, Liang Yunpei, Yuan Shijun. Simulation study on distribution regularity of fore abutment pressure in different mining thickness. Mining Safety\& Environmental Protection, 2008, 35(2):17-21.

[7]Xie Fuxing, Zhang Zhaoqian, Cui Kai. Advanced abutment pressure distribution rule and stress summit location in mining field with large mining-height. Coal Mining Technology, 2013, 18(1):80-83.

[8]Niu Jianzhong. Distribution of large mining height abutment pressure ahead of the short wall face. Shandong Coal Science and Technology, 2014, 12:26-30
[9]Wu Hai, Zhang Nong, Wang Weijun, Zhao Yiming, Cao Peng. Characteristics of deformation and stress distribution of small coal pillars under leading abutment pressure. International Journal of Mining Science and Technology, 2015, 25: 921-926.

[10] Liu Jinhai, Jiang Fuxing, Feng Tao. Numerical Simulation of Abutment Pressure Distribution of C-shaped Stope. Rock and Soil Mechanics, 2010, 31(12):4011-4015.

[11] Liu Changyou, Huang Bingxiang, Meng Xiangjun, Yang Peiju, Chen Longgao. Research on abutment pressure distribution law of overlength isolated fully-mechanized top coal caving face. Chinese Journal of Rock Mechanics and Engineering, 2007, 26(S): 2761-2766.

[12]Xu Wenquan, Wang Enyuan, Shen Rongxi, Song Dazhao, Zhang Jingmin. Distribution pattern of front abutment pressure of fully-mechanized working face of soft coal isolated island. International Journal of Mining Science and Technology, 2012, 22:279-284.

[13]Xia Yongxue, Lan Hang, Mao Debing, Pan Junfeng. Study of the lead abutment pressure distribution base on microseismic monitoring. Journal of China University of Mining \& Technology, 2011, 40(6):868-873.

[14]L. Jing, J.A. Hudson. Numerical methods in rock mechanics. International Journal of Rock Mechanics \& Mining Sciences, 2002, 39:409-427.

[15]Zhang Xiantang, Wang Hongli, Zhou Hongmin. Application of FLAC ${ }^{3 \mathrm{D}}$ to forecast water flow in subsea tunnel, Rock and Soil Mechanics, 2008,29:258-262.

[16]Shi Yuanwei, Zhang Shengtao, Yin Shikui, Bing Susheng, Li Congxin. The strata control technology for deep coal mining at home and abroad. Beijing, China Coal Industry Publishing Home, 2009.

[17]Xue Cheng, Zhao Jianfeng, Wang Gangsheng. Study on pilot roof support pressure distribution law of coal mining face in dongqu mine. Coal Science and Technology, 2011, 39(6):9-11.

[18]Qian Minggao, Shi Pingwu, Xu Jialin. Mining Pressure and Strata Control. Xuzhou, China University of Science and Technology Press, 2010.

[19] Zhang Xiantang, Li Xiaolong, Wang Hongli, Zhou Hongmin. Based on numerical simulation to investigate the process of gas explosion accident in coal mine. Advanced Materials Research, 2012,549:936-940.

[20]Itasca Consulting Group,Inc.. Fast Language Analysis of continua in 2 dimensions, version 5.0, user's mannual[R]. Itasca Consulting Group, Inc., 2005. 